MATHEMATICS OF COMPUTATION

Volume 72, Number 242, Pages 939-940

S 0025-5718(02)01450-3

Article electronically published on May 15, 2002

\title{
THE FIRST KNOWN TYPE $(7,1)$ AMICABLE PAIR
}

\author{
MARIANO GARCIA
}

\begin{abstract}
Amicable pairs of type $(i, 1)$ have been the object of special search partly because they breed other amicable pairs. Examples exist for $i=$ $2,3,4,5$, and 6 . In this paper we exhibit the first known type $(7,1)$ pair.
\end{abstract}

The natural numbers $M$ and $N$ are amicable if $\sigma(M)=\sigma(N)=M+N$, where $\sigma(M)$ represents the sum of the positive divisors of $M$. An amicable pair is of type $(i, 1)$ if $M=E \cdot p_{1} \cdot p_{2} \ldots p_{i}$ and $N=E \cdot q$, where $E$ is the greatest common factor of $M$ and $N$, and $q$ and each $p_{j}$ are different primes not dividing $E$ for $j=1,2, \ldots i$.

Type $(i, 1)$ amicable pairs have been the object of special investigation and search [1], 2], 3], [5]. Often it is possible to construct other amicable pairs from them 6]. Also, until Euler's time [4, the only three known amicable pairs were of type $(2,1)$. One of these is the smallest existing amicable pair $\left(2^{2} \cdot 5 \cdot 11,2^{2} \cdot 71\right)$, which was known to the Pythagoreans.

There are special web pages that contain information about $(i, 1)$ amicable numbers. Interested readers are referred to the collection of these pairs in the Internet 7 .

The first $(3,1)$ amicable pair was discovered by Poulet in 1941, the first $(4,1)$ pair by Lee in 1966, the first $(5,1)$ pair by te Riele in 1982 and the first $(6,1)$ pair by Pedersen in 1997. As of the writing of this article (March, 2001), there are 478 known amicable pairs of type $(2,1), 655$ pairs of type $(3,1), 248$ pairs of type $(4,1)$, 58 pairs of type $(5,1)$, and 7 pairs of type $(6,1)$. There are also 50 known amicable pairs of the so called exotic or irregular [7] type, which are closely related to $(i, 1)$ pairs as defined above.

The author has just found the first known type $(7,1)$ amicable pair, which follows:

$M=2 \cdot 5^{2} \cdot 19 \cdot p_{1} \cdot p_{2} \cdot p_{3} \cdot p_{4} \cdot p_{5} \cdot p_{6} \cdot p_{7}$,

$N=2 \cdot 5^{2} \cdot 19 \cdot q$

where

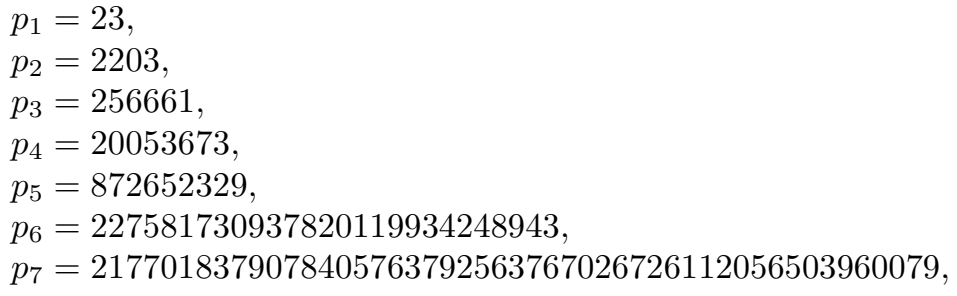

Received by the editor May 21, 2001.

2000 Mathematics Subject Classification. Primary 11A25.

Key words and phrases. Amicable pairs. 
and

$q=\left(p_{1}+1\right) \cdot\left(p_{2}+1\right) \cdot\left(p_{3}+1\right) \cdot\left(p_{4}+1\right) \cdot\left(p_{5}+1\right) \cdot\left(p_{6}+1\right) \cdot\left(p_{7}+1\right)-1$, which is a prime. Each member of the pair has 105 digits.

We were aided considerably in our search by the following unpublished theorem due to te Riele, that has been used by several researchers to discover $(i, 1)$ amicable pairs.

Theorem. Let $E$ and $M$ be two relatively prime natural numbers such that $\sigma(E)$. $\sigma(M)=E \cdot(M+\sigma(M)-1)$. Then $E \cdot M \cdot p \cdot q$ and $E \cdot[\sigma(M) \cdot(p+1) \cdot(q+1)-1]$ constitute an amicable pair, provided $p, q$ and $\sigma(M) \cdot(p+1) \cdot(q+1)-1$ are prime numbers not dividing $E$ or $M$ and satisfying the equation

$$
(p-M+1) \cdot(q-M+1)=M \cdot(M-1)+1 .
$$

The author has obtained several amicable pairs generated 6] from the type $(7,1)$ pair. These numbers are of the form $\left(2 \cdot 5^{2} \cdot 19 \cdot\right.$ product of eight primes, $2 \cdot 5^{2} \cdot 19 \cdot$ product of two primes). There are 122444006400 cases to be considered in the generation algorithm, and with the facilities available to us, it would take the author several years to obtain all the amicable pairs arising in this manner. As an illustration, one of the pairs obtained is the following:

$M=2 \cdot 5^{2} \cdot 19 \cdot p_{1} \cdot p_{2} \cdot p_{3} \cdot p_{4} \cdot p_{5} \cdot p_{6} \cdot p_{7} \cdot p_{8}$,

$N=2 \cdot 5^{2} \cdot 19 \cdot q_{1} \cdot q_{2}$

where $p_{1}, p_{2}, p_{3}, p_{4}, p_{5}, p_{6}$, and $p_{7}$ are the same as in our type $(7,1)$ pair, $q_{1}$ is a prime with 102 digits, and $p_{8}$ and $q_{2}$ are primes with 125 digits each.

\section{REFERENCES}

1. W. Borho and H. Hoffmann, Breeding amicable numbers in abundance, Math. Comp. 46 (1986), 281-293. MR 87c:11003

2. P. Costello, Amicable pairs of the form $(i, 1)$, Math. Comp. 56 (1991), 859-865. MR 91k:11009

3. E. B. Escott, Amicable numbers, Scripta Math. 12 (1946), 61-72. MR 8:135a

4. L. Euler, De numeris amicabilibus, Opuscula varii argumenti (1750), 23-107.

5. E. J. Lee and J. S. Madachy, The history and discovery of amicable numbers, J. Recr. Math. 5 (1972), Part 1, 77-93, Part 2, 153-173, Part 3, 231-249. MR 56:5165a; MR 56:5165b; MR 56:5165c errata MR 56:5165d errata MR 56:5165e

6. H. J. J. te Riele, On generating new amicable pairs from given amicable pairs, Math. Comp. 42 (1984), 219-223. MR 85d:11107

7. J. Pedersen, Various amicable pair lists and statistics, http://www.vejlehs.dk/staff/ jmp/aliquot/apstat.htm.

Department of Mathematics, Touro College, 27 West 23rd Street, New York, New YORK 10010 